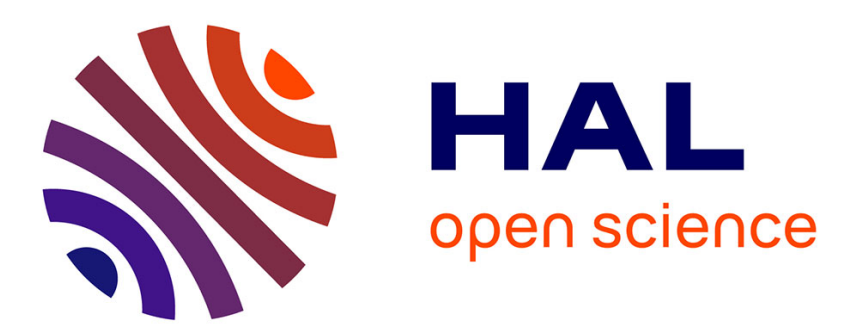

\title{
Adaptive scheme for pathological oscillations disruption in a delayed neuronal population model
}

Jakub Orlowski, Antoine Chaillet, Mario Sigalotti, Alain Destexhe

\section{To cite this version:}

Jakub Orlowski, Antoine Chaillet, Mario Sigalotti, Alain Destexhe. Adaptive scheme for pathological oscillations disruption in a delayed neuronal population model. 57th IEEE Conference on Decision and Control (CDC 2018), Dec 2018, Miami Beach, United States. hal-01956472

HAL Id: hal-01956472

https://hal-centralesupelec.archives-ouvertes.fr/hal-01956472

Submitted on 15 Dec 2018

HAL is a multi-disciplinary open access archive for the deposit and dissemination of scientific research documents, whether they are published or not. The documents may come from teaching and research institutions in France or abroad, or from public or private research centers.
L'archive ouverte pluridisciplinaire HAL, est destinée au dépôt et à la diffusion de documents scientifiques de niveau recherche, publiés ou non, émanant des établissements d'enseignement et de recherche français ou étrangers, des laboratoires publics ou privés. 


\title{
Adaptive scheme for pathological oscillations disruption in a delayed neuronal population model
}

\author{
Jakub Orłowski, Antoine Chaillet, Mario Sigalotti, and Alain Destexhe
}

\begin{abstract}
Motivated by improved ways to disrupt brain oscillations linked to Parkinson's disease, we propose an adaptive output feedback strategy for the stabilization of nonlinear timedelay systems evolving on a bounded set. To that aim, using the formalism of input-to-output stability (IOS), we first show that, for such systems, internal stability guarantees robustness to exogenous disturbances. We then use this feature to establish a general result on scalar adaptive output feedback of time-delay systems inspired by the " $\sigma$-modification" strategy. We finally apply this result to a delayed neuronal population model and assess numerically the performance of the adaptive stimulation.
\end{abstract}

\section{INTRODUCTION}

Parkinsonian symptoms correlate with sustained oscillatory activity in deep brain regions known as basal ganglia [9]. Deep brain stimulation (DBS) is an efficient symptomatic treatment of parkinsonian symptoms that consists in delivering high-frequency electrical impulses to those regions through permanently implanted electrodes [1]. So far, this electrical stimulation is delivered in open loop, with no consideration of the real-time activity of the patient's brain. Several theoretical and experimental approaches have aimed at developing closed-loop DBS strategies [3]. In particular, it has been shown that a simple proportional control successfully disrupts these pathological oscillations in a population model of the brain structures involved [7], [4]. These strategies require large feedback gains, whose precise values depend on the system's parameters. These parameters are not easily accessible to measurements and may vary in time. These issues plead for the development of adaptive control strategies, in which the stimulation gain would automatically adapt to a convenient value. This would, in turn, lead to lower energy consumption and a more parsimonious stimulation than constant high-gain policies.

The main goal of this paper is to develop an adaptive stimulation strategy to disrupt pathological brain oscillations. Adaptive control for high-gain stabilizable systems can be derived through classical techniques such as [2], [18] (see [20] for a survey). Many approaches have been proposed to increase robustness of such approaches, including the " $\sigma$ modification" proposed in [12], which adds a dissipative

J.O. is with Lab. des Signaux et Systèmes, CentraleSupélec, Univ. Paris Saclay, France jakub.orlowskiel2s.centralesupelec.fr. A.C. is with Lab. des Signaux et Systèmes, CentraleSupélec, Univ. Paris Saclay, France and with Institut Universitaire de France antoine.chailletecentralesupelec.fr. A.D. is with UNIC, CNRS, France destexhedunic.cnrs-gif.fr. M.S. is with Sorbonne Univ., Univ. Paris-Diderot SPC, CNRS, INRIA, Lab. Jacques-Louis Lions, équipe Cage, France mario.sigalottieinria.fr. Supported by the E.C. (Human Brain Project H2020-785907) to A.D., and by the iCODE project funded by the IDEX Paris-Saclay, ANR-11-IDEX-0003-02. term in the gain adaptation law, as well as the " $\lambda$-tracking", proposed in [11] and applied to linear infinite-dimensional systems in [10], which increases robustness with respect to external noise by guaranteeing that the tracking error converges to a set of a specified radius. In the context of parkinsonian brain oscillations, the strong uncertainty and possible evolution in time of the model parameters naturally lead to the application of the aforementioned $\sigma$-modification.

A key feature to be taken into account in the control design is the presence of delays in the dynamics, representing the non-instantaneous communication between neurons due to axonal propagation. Delays are believed to play a crucial role in the onset of pathological oscillations [19]. This peculiarity requires to adapt existing adaptive control strategies to timedelay systems. To that aim, we take advantage of another specificity of neuronal dynamics, namely their evolution over a bounded set, as neuronal activity cannot overpass certain limits due to neurophysiological considerations.

In order to prove the efficiency of the $\sigma$-modification adaptative strategy for nonlinear time-delay systems, we rely on the framework of input-to-output stability (IOS), originally introduced in [22] in a finite-dimensional context and extended to delay systems in [16]. We show that, for systems evolving on a bounded set, this property boils down to asymptotic stability in the absence of disturbances. This observation allows us to reduce the problem to controlling a disturbance-free system with an adaptive control whose gain never decreases in time (unlike the $\sigma$-modification). After presenting a general adaptive control strategy for nonlinear time-delay systems with bounded dynamics, we apply this methodology to the neuronal population model of [19], which already served as a basis for the developments in [7], and assess its performance through numerical simulations.

Notation. Given $x \in \mathbb{R}^{n},|x|$ denotes its Euclidean norm. Given a compact set $I \subset \mathbb{R}$ and a measurable signal $u$ : $I \rightarrow \mathbb{R}^{m},\|u\|:=$ ess $\sup _{t \in I}|u(t)|$. Given $b>a, u_{[a ; b]}$ : $[a ; b] \rightarrow \mathbb{R}^{m}$ denotes the function defined as $u_{[a ; b]}(t)=u(t)$ for all $t \in[a ; b]$. Given $X \subset \mathbb{R}^{n}, C(I, X)$ denotes the set of all continuous functions $\phi: I \rightarrow X$ and, given $\bar{\delta}>0$, $\mathcal{C}:=C([-\bar{\delta} ; 0], \mathbb{R})$. Given a signal $x:[-\bar{\delta},+\infty) \rightarrow \mathbb{R}^{n}$ and a time $t \geq 0, x_{t} \in \mathcal{C}^{n}$ denotes the history function: $x_{t}(s):=$ $x(t+s)$ for all $s \in[-\bar{\delta} ; 0]$. A function $\alpha: \mathbb{R}_{\geq 0} \rightarrow \mathbb{R}_{\geq 0}$ is said to be of class $\mathcal{P D}$ if it is continuous and positive definite. $\alpha \in \mathcal{K}$ if $\alpha \in \mathcal{P D}$ and it is increasing. $\alpha \in \mathcal{K}_{\infty}$ if $\alpha \in \mathcal{K}$ and it is unbounded. A function $\beta: \mathbb{R}_{\geq 0} \times \mathbb{R}_{\geq 0} \rightarrow \mathbb{R}_{\geq 0}$ is of class $\mathcal{K} \mathcal{L}$ if $\beta(\cdot, t) \in \mathcal{K}$ for each $t \in \mathbb{R}_{>0}$ and, for each $s \in \mathbb{R}_{\geq 0}, \beta(s, \cdot)$ is continuous, non-increasing and tends to zero as its argument tends to infinity. 


\section{II. $y$-GAS AND IOS}

We start by recalling and adapting results on the stability of input-free systems and their robustness with respect to disturbances, in a time-delay context. Let $\mathcal{X}$ be an open subset of $\mathcal{C}^{n}$ for the topology of the uniform convergence. Let $U$ be an open subset of $\mathbb{R}$ containing the origin, and $\mathcal{U}$ be the set of all measurable and locally essentially bounded $u: \mathbb{R}_{\geq 0} \rightarrow U$. Consider the perturbed time-delay system:

$$
\dot{x}(t)=f\left(x_{t}, u(t)\right), \quad y(t)=h\left(x_{t}\right),
$$

where $x_{t} \in \mathcal{X}$ is the state, $u \in \mathcal{U}^{m}$ is the applied input, and $y \in C\left(\mathbb{R}_{\geq 0}, \mathbb{R}^{l}\right)$ is an output of interest. We start by recalling Driver's formulation of the upper-right Dini derivative [6]. Given any continuous function $V: \mathcal{X} \rightarrow \mathbb{R}$, its Driver's derivative $D^{+} V$ along the solutions of (1) is defined, for all $\phi \in \mathcal{X}$ and all $v \in U^{m}$, as

$$
D_{(1)}^{+} V(\phi, v):=\limsup _{h \rightarrow 0^{+}} \frac{V\left(\phi_{h, v}^{\star}\right)-V(\phi)}{h},
$$

where, for all $h \in(0 ; \bar{\delta}), \phi_{h, v}^{\star} \in \mathcal{X}$ is defined as

$$
\phi_{h, v}^{\star}(s):= \begin{cases}\phi(s+h) & \text { if } s \in[-\bar{\delta} ;-h) \\ \phi(0)+f(\phi, v)(s+h) & \text { if } s \in[-h ; 0] .\end{cases}
$$

It was shown in [21] that, if $V$ is Lipschitz on any bounded set of $\mathcal{C}^{n}$, then Driver's derivative of $V$ computed at $\left(x_{t}, u(t)\right)$ coincides, at almost every $t$ where $x_{t}$ is defined, with the upper-right Dini derivative of $t \mapsto V\left(x_{t}\right)$, that is,

$$
D_{(1)}^{+} V\left(x_{t}, u(t)\right)=\limsup _{\tau \rightarrow 0^{+}} \frac{V\left(x_{t+\tau}\right)-V\left(x_{t}\right)}{\tau}, \quad \text { a.e. }
$$

\section{A. Characterizations of $y-G A S$}

Let us first consider an input-free version of (1):

$$
\dot{x}(t)=f\left(x_{t}\right), \quad y(t)=h\left(x_{t}\right),
$$

where $y(t) \in \mathbb{R}^{l}$ denotes an output for which we want to ensure a desirable behavior. We assume the following.

Assumption 1 There exists a bounded open forwardinvariant set $\mathcal{X} \subset \mathcal{C}^{n}$ for (4), the functionals $f: \mathcal{C}^{n} \rightarrow \mathbb{R}^{n}$ and $h: \mathcal{C}^{n} \rightarrow \mathbb{R}^{l}$ are Lipschitz on $\mathcal{X}$, and $f(0)=0$.

The above regularity assumptions are standard and ensure existence and uniqueness of solutions [8, Section 2.6, p. 55]. We also impose that solutions evolve on a bounded invariant set as this assumption greatly simplifies the analysis and it is naturally satisfied in the neuroscience applications considered here (see Section IV). Note that this boundedness assumption in turn ensures forward completeness of (4).

For the needs of this paper, we will study global asymptotic output-stability as defined in the following sense.

Definition 1 ( $y$-GAS) We say that system (4) is globally asymptotically $y$-stable ( $y$-GAS) on $\mathcal{X}$ if there exists $\beta \in \mathcal{K} \mathcal{L}$ such that, for all $x_{0} \in \mathcal{X}$, its solution satisfies

$$
\left|h\left(x_{t}\right)\right| \leq \beta\left(\left\|x_{0}\right\|, t\right), \quad \forall t \geq 0 .
$$

This stability notion is strongly related to "stability with respect to two measures" employed in [17], [23], to "partial stability" studied in [24], as well as to output stability concepts [22] that were more recently extended to timedelay systems [15], [16], [13]. It imposes that the output $y$ eventually converges to the origin, with a transient overshoot that may depend on the magnitude of whole initial state $x_{0}$.

This output-stability can be established through the following Lyapunov-Krasovskii characterizations.

\section{Theorem 1 ( $y$-GAS characterizations) Under Assumption} 1, the following statements are equivalent:

1) System (4) is y-GAS on $\mathcal{X}$.

2) There exist a Lipschitz functional $V: \mathcal{X} \rightarrow \mathbb{R}_{\geq 0}$ and $\underline{\alpha}, \bar{\alpha} \in \mathcal{K}_{\infty}$ such that, for all $\phi \in \mathcal{X}$,

$$
\begin{gathered}
\underline{\alpha}(|h(\phi)|) \leq V(\phi) \leq \bar{\alpha}(\|\phi\|) \\
D_{(4)}^{+} V(\phi) \leq-V(\phi) .
\end{gathered}
$$

3) There exist a Lipschitz functional $V: \mathcal{X} \rightarrow \mathbb{R}_{\geq 0}$ and $\underline{\alpha}, \bar{\alpha}, \alpha \in \mathcal{K}_{\infty}$ such that, for all $\phi \in \mathcal{X}$,

$$
\begin{gathered}
\underline{\alpha}(|h(\phi)|) \leq V(\phi) \leq \bar{\alpha}(\|\phi\|) \\
D_{(4)}^{+} V(\phi) \leq-\alpha(|h(\phi)|) .
\end{gathered}
$$

Proof: The equivalence between Items 1) and 2) is a direct consequence of [16, Theorem 3.3]. More precisely, all the requirements $(\mathrm{S} 1)-(\mathrm{S} 8)$ in that paper are satisfied under Assumption 1, and the property referred to as "almost Lipschitz" in that paper turns out to be "Lipschitz on bounded sets" in the present time-invariant context. Moreover, Item 2) readily implies Item 3) as (5)-(6) imply that $D_{(4)}^{+} V(\phi) \leq$ $-\underline{\alpha}(|h(\phi)|)$. Finally, the fact that Item 3) implies Item 1) was proven in [24, Theorem 6.2.1]. Although that reference makes only use of " $\varepsilon-\delta$ " stability definitions, the $\mathcal{K} \mathcal{L}$ formulation employed in Definition 1 can be recovered using similar reasonings as in the proof of [14, Lemma 3.4].

\section{B. IOS for 0-y-GAS systems evolving on bounded sets}

We now go back to the perturbed system (1) to study the impact of disturbances on output-stability. To that aim, recall the notion of input-to-output stability (IOS), originally introduced in [22] in a finite-dimensional context and extended in [16], [13] to time-delay systems.

Definition 2 (IOS) System (1) is said to be input-to-output stable (IOS) on $\mathcal{X}$ and $\mathcal{U}^{m}$ if there exist $\beta \in \mathcal{K} \mathcal{L}$ and $\gamma \in \mathcal{K}_{\infty}$ such that, for all $x_{0} \in \mathcal{X}$ and all $u \in \mathcal{U}^{m}$, its solution satisfies

$$
\left|h\left(x_{t}\right)\right| \leq \beta\left(\left\|x_{0}\right\|, t\right)+\gamma\left(\left\|u_{[0 ; t]}\right\|\right), \quad \forall t \geq 0 .
$$

IOS not only imposes $y$-GAS in the absence of disturbances, but also that, in their presence, the output eventually converges to a neighborhood of the origin whose size is "proportional" to their magnitude.

Here also, we assume that the system evolves on a bounded set for all considered initial states and disturbances. 
Assumption 2 There exists a bounded open set $\mathcal{X} \subset \mathcal{C}^{n}$ which is forward-invariant for (1) for any $u \in \mathcal{U}^{m}$. Moreover, the functionals $f: \mathcal{C}^{n} \times U^{m} \rightarrow \mathbb{R}^{n}$ and $h: \mathcal{C}^{n} \rightarrow \mathbb{R}^{l}$ are Lipschitz on $\mathcal{X} \times U^{m}$ and $\mathcal{X}$ respectively, and $f(0,0)=0$.

The above assumption requires that solutions of the perturbed system (1) evolve on the bounded set $\mathcal{X}$ no matter what the applied input $u \in \mathcal{U}^{m}$. Here again this assumption is naturally satisfied for the relevant neuroscience applications.

Definition 3 (0-y-GAS) System (1) is said to be 0-y-GAS if the following input-free system is $y$-GAS:

$$
\dot{x}(t)=f\left(x_{t}, 0\right), \quad y(t)=h\left(x_{t}\right) .
$$

Clearly, IOS implies 0-y-GAS. Based on Theorem 1, we can show that these two properties are actually equivalent for systems evolving in a bounded set, as stated next.

Theorem 2 (0-y-GAS $\Leftrightarrow$ IOS, on bounded sets) Under Assumption 2, system (1) is IOS on $\mathcal{X}$ and $\mathcal{U}^{m}$ if and only if the input-free system (10) is $y$-GAS on $\mathcal{X}$.

In the above statement, the fact that solutions evolve in a bounded set is crucial. Without this assumption, IOS is strictly more conservative that $0-y$-GAS. A similar result was obtained in [25] for systems that are globally exponentially stable in the absence of disturbances. That result does not require that solutions evolve on a bounded set, but rather imposes that $f$ is globally Lipschitz and that there exist $c \geq 0$ and $p \in[0 ; 1)$ such that $|f(\phi, v)-f(\phi, 0)| \leq$ $c \max \left\{\|\phi\|^{p}, 1\right\}|v|$ for all $\phi \in \mathcal{C}^{n}$ and all $v \in \mathbb{R}^{m}$.

Due to space limitation, the proof of this theorem is not included but proceeds as follows. We only prove that $0-y$ GAS implies IOS, as the converse is straightforward. From Theorem 1 we get the existence of a Lyapunov-Krasovskii functional $V$ satisfying (5)-(6) along the disturbance-free system. Using the properties of the Driver's derivative, Lipschitz continuity of $V$ and $f$ (Assumption 4), and the fact that the system evolves on a bounded set, we compute the derivative of $V$ along the solutions of the perturbed system (1) to get that $D_{(1)}^{+} V(\phi, v) \leq-V(\phi)+\ell_{V} \ell_{f}|v|$ for all $\phi \in \mathcal{X}$ and all $v \in U^{m}$, where $\ell_{V}$ is the Lipschitz constant of $V$ on $\mathcal{X}$ and $\ell_{f}$ is the Lipschitz constant of $f$ on $\mathcal{X} \times U^{m}$. This fulfills condition d) of [16, Thm. 3.3], establishing IOS.

\section{ADAPTIVE GAIN FOR TIME-DELAY SYSTEMS}

We now make use of these results for adaptive control. Consider the following class of time-delay control systems:

$$
\begin{aligned}
\dot{x}(t) & =f\left(x_{t}, \mu(t), u(t)\right) \\
z(t) & =g\left(x_{t}\right),
\end{aligned}
$$

where $x_{t} \in \mathcal{C}^{n}$ is the state and $u \in \mathcal{U}^{m}$ represents a disturbance acting on the system. $\mu(t) \in \mathbb{R}$ denotes the scalar control input. $z(t) \in \mathbb{R}$ denotes the scalar output available for measurements. The adaptive control we propose here is based on the " $\sigma$-modification" originally introduced in [12] for linear systems with no delay: $\mu$ is defined in terms of an additional scalar variable $\theta$ as follows:

$$
\begin{aligned}
\mu(t) & =-\theta(t) z(t) \\
\dot{\theta}(t) & =\kappa(z(t))-\varepsilon \theta(t),
\end{aligned}
$$

where $\varepsilon \geq 0$ is a tunable parameter and $\kappa: \mathbb{R}_{\geq 0} \rightarrow \mathbb{R}_{\geq 0}$ is a locally Lipschitz function to be chosen. As illustrated in Section IV-B, the choice of $\varepsilon$ and $\kappa$ can be guided by the sought closed-loop performance. We assume the following.

Assumption 3 There exists a bounded open set $\mathcal{X} \subset \mathcal{C}^{n}$ such that for every $u \in \mathcal{U}^{m}$, every continuous $\mu: \mathbb{R}_{\geq 0} \rightarrow \mathbb{R}$, and every $\varepsilon \geq 0$, the set $\mathcal{X}$ is forward invariant for equation (11a). The functionals $f: \mathcal{C}^{n} \times \mathbb{R} \times U^{m} \rightarrow \mathbb{R}^{n}$ and $g: \mathcal{C}^{n} \rightarrow$ $\mathbb{R}$ are Lipschitz on bounded sets, and $f(0,0,0)=0$.

We also assume that the origin of this plant is globally exponentially stabilizable by high-gain proportional feedback in the absence of disturbances $u$, as stated in the following.

Assumption 4 (High-gain stabilizability) There exist a Lipschitz functional $V: \mathcal{X} \rightarrow \mathbb{R}_{\geq 0}$, positive constants $\underline{a}$, $\bar{a}$ and $a$, and a positive gain $\theta^{*}$ such that, for all $\phi \in \mathcal{X}$,

$$
\underline{a}|\phi(0)|^{2} \leq V(\phi) \leq \bar{a}\|\phi\|^{2},
$$

and, for all fixed $\theta \geq \theta^{*}$, Driver's derivative of $V$ satisfies

$$
D_{(15)}^{+} V(\phi, \theta) \leq-a|\phi(0)|^{2}
$$

along the solutions of the closed-loop system

$$
\dot{x}(t)=f\left(x_{t},-\theta z(t), 0\right), \quad z(t)=g\left(x_{t}\right) .
$$

Our main result is as follows.

Theorem 3 (Robust stability with adaptive gain) Let $\kappa$ : $\mathbb{R} \rightarrow \mathbb{R}_{\geq 0}$ be any locally Lipschitz function satisfying

$$
\kappa(s) \geq \kappa_{0}|s|, \quad \forall s \in \mathbb{R},
$$

for some $\kappa_{0}>0$ and $\kappa(g(0))=0$. Then, under Assumptions 3 and 4 , there exist $\beta \in \mathcal{K} \mathcal{L}, \gamma \in \mathcal{K}_{\infty}$, and $\vartheta>\theta^{*}$ such that, for all $\left(x_{0}, \theta_{0}\right) \in \mathcal{X} \times\left[0 ; \theta^{*}\right]$, all $u \in \mathcal{U}^{m}$, and all $\varepsilon \geq 0$, the solution of (11) in closed-loop with (12) satisfies

$$
\begin{aligned}
|x(t)| & \leq \beta\left(\left\|x_{0}\right\|+\left|\theta_{0}-\theta^{*}\right|, t\right)+\gamma\left(\varepsilon+\left\|u_{[0 ; t]}\right\|\right) \\
\theta(t) & \in[0 ; \vartheta], \quad \forall t \geq 0 .
\end{aligned}
$$

The above statement contains several ingredients. First, in the absence of disturbances $(u \equiv 0)$, practical stability is achieved in the sense that the steady-state error on $x$ can be arbitrarily reduced by picking $\varepsilon$ sufficiently small. The transient overshoot is "proportional" to the magnitude of the initial state and to the distance between $\theta_{0}$ and $\theta^{*}$ (this distance being small if we initialize the control algorithm with a good guess of $\theta^{*}$ ). For $\varepsilon=0$, this steady-state error is zero, meaning that $x(t)$ converges to the origin as $t$ tends to infinity. The use of a positive $\varepsilon$ allows to avoid parameter drift in presence of disturbances [12], as certified by (18). More crucially, $\varepsilon>0$ allows to react to possible variations of 
the model parameters, as detailed in Section IV-B. Finally, in the presence of disturbances, the state is guaranteed to converge to a neighborhood of the origin, whose size is "proportional" to the magnitude of the applied disturbance and the chosen value of the parameter $\varepsilon$.

Proof: We see the system as a perturbed version of

$$
\dot{x}(t)=f\left(x_{t},-\theta(t) g\left(x_{t}\right), 0\right), \quad \dot{\theta}(t)=\kappa\left(g\left(x_{t}\right)\right) .
$$

In other words, we see both $u$ and $\varepsilon$ as perturbations. In view of Theorem 1, IOS of the closed-loop system can be derived based on the output-stability of (19). Let $\theta^{*}$ be as in Assumption 4 and let $\tilde{\theta}:=\theta-\theta^{*}$. Then (19) reads

$$
\begin{aligned}
& \dot{x}(t)=f\left(x_{t},-\left(\tilde{\theta}(t)+\theta^{*}\right) g\left(x_{t}\right), 0\right) \\
& \dot{\tilde{\theta}}(t)=\kappa\left(g\left(x_{t}\right)\right) .
\end{aligned}
$$

The results of Section II do not readily apply to this system since $\tilde{\theta}$ does not necessarily evolve in a bounded set (because $\left.\kappa\left(g\left(x_{t}\right)\right) \geq 0\right)$. However, we can show that there exists $\bar{\theta}>0$ such that $\tilde{\tilde{\theta}}(t) \in\left[-\theta^{*}, \bar{\theta}\right]$ for all $t \geq 0$ and all $\tilde{\theta}_{0} \in\left[-\theta^{*}, 0\right]$. Hence, we can consider the modified dynamics:

$$
\begin{aligned}
\dot{x}(t) & =f\left(x_{t},-\left(\tilde{\theta}(t)+\theta^{*}\right) g\left(x_{t}\right), 0\right) \\
\dot{\tilde{\theta}}(t) & =\kappa\left(g\left(x_{t}\right)\right)-\max \{\tilde{\theta}(t)-\bar{\theta} ; 0\}(\tilde{\theta}(t)-\bar{\theta}) .
\end{aligned}
$$

This modified system (21) does possess an invariant set, even in presence of disturbances, as stated next. We express the invariance in terms of the history function for $\theta$ (even though no delays apply to it) in order to apply later to the augmented state variable $(x, \theta)$ the results of Section II.

Claim 1 Let $\bar{\kappa}$ be any positive constant such that $\kappa \circ g(\phi) \leq$ $\bar{\kappa}$ for all $\phi \in \mathcal{X}$, as ensured by Assumption 3, and let

$$
\Theta:=C\left([-\bar{\delta} ; 0],\left(-2 \theta^{*} ; 2 \bar{\theta}+\sqrt{\bar{\kappa}}\right)\right) \subset \mathcal{C} .
$$

Then, for every $u \in \mathcal{U}^{m}$ and $\varepsilon \geq 0$, the bounded open set $\mathcal{X} \times \Theta$ is invariant for

$$
\begin{aligned}
\dot{x}(t)= & f\left(x_{t},-\left(\tilde{\theta}(t)+\theta^{*}\right) g\left(x_{t}\right), u\right) \\
\dot{\tilde{\theta}}(t)= & \kappa\left(g\left(x_{t}\right)\right)-\varepsilon\left(\tilde{\theta}(t)+\theta^{*}\right) \\
& -\max \{\tilde{\theta}(t)-\bar{\theta} ; 0\}(\tilde{\theta}(t)-\bar{\theta}) .
\end{aligned}
$$

Proof: In view of Assumption 3, the claim readily follows by observing that $\dot{\tilde{\theta}}(t) \leq 0$ whenever $\tilde{\theta}(t) \geq \bar{\theta}+\sqrt{\bar{\kappa}}$ (and in particular for $\tilde{\theta}(t) \geq 2 \bar{\theta}+\sqrt{\bar{\kappa}}$ ) and that $\dot{\tilde{\theta}}(t) \geq 0$ whenever $\tilde{\theta}(t) \leq \bar{\theta}$ (and in particular for $\tilde{\theta}(t) \leq-2 \theta^{*}$ ).

The claim in particular proves (18) with $\vartheta=2 \bar{\theta}+\sqrt{\bar{\kappa}}+\theta^{*}$.

Based on this, we now proceed to show that (21) is IOS on $\mathcal{X} \times \Theta$ with output $y(t)=x(t)$. To that end, let $V$ be as in Assumption 4 and consider the functional $W: \mathcal{X} \times \mathcal{C} \rightarrow \mathbb{R}_{\geq 0}$ defined as

$$
W(\phi, \psi)=V(\phi)+\frac{p}{2} \min \{\psi(0) ; 0\} \psi(0),
$$

where $p$ denotes a positive constant to be chosen later. Then $W$ is Lipschitz on $\mathcal{X} \times \Theta$ and, for all $(\phi, \psi) \in \mathcal{X} \times \mathcal{C}$,

$$
\underline{a}|\phi(0)|^{2} \leq W(\phi, \psi) \leq\left(\bar{a}+\frac{p}{2}\right)\|(\phi, \psi)\|^{2} .
$$

Moreover, if $\psi(0) \geq 0$ (corresponding to $\theta(t) \geq \theta^{*}$ ), then $W(\phi, \psi)=V(\phi)$ and it holds from Assumption 4 that

$$
D_{(20)}^{+} W(\phi, \psi) \leq-a|\phi(0)|^{2}, \quad \forall \psi(0) \geq 0 .
$$

Consider now the case when $\psi(0)<0$. Then $W(\phi, \psi)=$ $V(\phi)+\frac{p}{2} \psi(0)^{2}$. It follows that

$$
D_{(20)}^{+} W(\phi, \psi)=D_{(20)}^{+} V(\phi, \psi)+p \psi(0) \kappa(g(\phi)) .
$$

Moreover, from (2),

$$
D_{(20)}^{+} V(\phi, \psi)=\limsup _{\tau \rightarrow 0^{+}} \frac{1}{\tau}\left(V\left(\phi_{\tau, \psi}^{\star}\right)-V(\phi)\right),
$$

where, in view of (3), $\phi_{\tau, \psi}^{\star}(s)=\phi(s+\tau)$ for all $s \in$ $[-\bar{\delta} ;-\tau)$ and, for all $s \in[-\tau ; 0]$,

$$
\phi_{\tau, \psi}^{\star}(s)=\phi(0)+f\left(\phi,-\left(\psi(0)+\theta^{*}\right) g(\phi), 0\right)(s+\tau) .
$$

Observe that, for all $(\phi, \psi) \in \mathcal{X} \times \mathcal{C}$ and all $\tau>0$ sufficiently small, it holds that $\phi_{\tau, \psi}^{\star} \in \mathcal{X}$. Let $\ell_{V}$ denote the Lipschitz constant of $V$ over the bounded set $\mathcal{X}$. Then, for all $(\phi, \psi) \in$ $\mathcal{X} \times \mathcal{C}$, it holds from (27) that

$$
\begin{gathered}
D_{(20)}^{+} V(\phi, \psi) \leq D_{(20)}^{+} V(\phi, 0)+\limsup _{\tau \rightarrow 0^{+}} \frac{1}{\tau}\left|V\left(\phi_{\tau, \psi}^{\star}\right)-V\left(\phi_{\tau, 0}^{\star}\right)\right| \\
\leq-a|\phi(0)|^{2}+\limsup _{\tau \rightarrow 0^{+}} \frac{\ell_{V}}{\tau}\left\|\phi_{\tau, \psi}^{\star}-\phi_{\tau, 0}^{\star}\right\|
\end{gathered}
$$

where we used (14) by observing that $D_{(20)}^{+} V(\phi, 0)$ is $D_{(19)}^{+} V(\phi, \psi)$ computed for $\theta=\theta^{*}$. Furthermore, since $\phi_{\tau, \psi}^{\star}$ and $\phi_{\tau, 0}^{\star}$ coincide over $[-\bar{\delta} ;-\tau]$, it holds that

$$
\begin{aligned}
& \left\|\phi_{\tau, \psi}^{\star}-\phi_{\tau, 0}^{\star}\right\|=\sup _{s \in[-\tau ; 0]}\left|\phi_{\tau, \psi}^{\star}(s)-\phi_{\tau, 0}^{\star}(s)\right|= \\
& \sup _{s \in[-\tau ; 0]}\left|f\left(\phi,-\left(\psi(0)+\theta^{*}\right) g(\phi), 0\right)-f\left(\phi,-\theta^{*} g(\phi), 0\right)\right|(s+\tau) .
\end{aligned}
$$

Let $G:=\left\{-\left(\psi(0)+\theta^{*}\right) g(\phi): \phi \in \mathcal{X}, \psi \in \Theta\right\}$ and let $\ell_{f}>0$ denote the Lipschitz constant of $f$ on $\mathcal{X} \times G \times\{0\}$, as ensured by Assumption 3. Then we get that

$\left\|\phi_{\tau, \psi}^{\star}-\phi_{\tau, 0}^{\star}\right\|=\sup _{s \in[-\tau ; 0]} \ell_{f}|\psi(0) g(\phi)|(s+\tau)=\ell_{f}|\psi(0) g(\phi)| \tau$.

Plugging this into (28), we obtain that $D_{(20)}^{+} V(\phi, \psi) \leq$ $-a|\phi(0)|^{2}+\ell_{V} \ell_{f}|\psi(0) g(\phi)|$. Thus, recalling that $\psi(0)<0$ and $\kappa(g(\phi)) \geq 0$, we get from (26) that

$$
\begin{aligned}
D_{(20)}^{+} W(\phi, \tilde{\theta}) & \leq-a|\phi(0)|^{2}-\left(p \kappa(g(\phi))-\ell_{V} \ell_{f}|g(\phi)|\right)|\psi(0)| \\
& \leq-a|\phi(0)|^{2}-\left(p \kappa_{0}-\ell_{V} \ell_{f}\right)|g(\phi)||\psi(0)|,
\end{aligned}
$$

where the last bound comes from (16). Therefore, by picking $p=\ell_{f} \ell_{V} / \kappa_{0}$, we obtain that, for all $(\phi, \psi) \in \mathcal{X} \times \Theta$,

$$
D_{(20)}^{+} W(\phi, \psi) \leq-a|\phi(0)|^{2}, \quad \forall \psi(0)<0 .
$$

Using (25), we conclude that, for all $(\phi, \psi) \in \mathcal{X} \times \Theta$, $D_{(20)}^{+} W(\phi, \tilde{\theta}) \leq-a|\phi(0)|^{2}$. Based on this, (24), and Claim 1, Theorem 1 ensures that there exists $\tilde{\beta} \in \mathcal{K} \mathcal{L}$ such that, for all $x_{0} \in \mathcal{X}$ and all $\tilde{\theta}_{0} \in\left(-2 \theta^{*} ; 2 \bar{\theta}+\sqrt{\bar{\kappa}}\right)$, the solution of $(21)$ satisfies $|x(t)| \leq \tilde{\beta}\left(\left\|x_{0}\right\|+\left|\tilde{\theta}_{0}\right|, t\right)$ for every $t \geq 0$. Theorem 2 , in turn, guarantees the existence of $\beta \in \mathcal{K} \mathcal{L}$ and $\gamma \in \mathcal{K}_{\infty}$ 
such that, for all $x_{0} \in \mathcal{X}$, all $\tilde{\theta}_{0} \in\left(-2 \theta^{*} ; 2 \bar{\theta}+\sqrt{\bar{\kappa}}\right)$ and all $u \in \mathcal{U}^{m}$, the solution of (21) satisfies

$$
|x(t)| \leq \beta\left(\left\|x_{0}\right\|+\left|\tilde{\theta}_{0}\right|, t\right)+\gamma\left(\varepsilon+\left\|u_{[0 ; t]}\right\|\right), \quad \forall t \geq 0 .
$$

We conclude that the same bound holds for the solutions of (20) starting within $\mathcal{X}$ and $\tilde{\theta}_{0} \in\left[-\theta^{*} ; 0\right]$. In particular, the solutions of (24) satisfy

$$
|x(t)| \leq \beta\left(\left\|x_{0}\right\|+\left|\theta_{0}-\theta^{*}\right|, t\right)+\gamma\left(\varepsilon+\left\|u_{[0 ; t]}\right\|\right), \quad \forall t \geq 0,
$$

for all $x_{0} \in \mathcal{X}$, all $\theta_{0} \in\left[0 ; \theta^{*}\right]$, and all $u \in \mathcal{U}^{m}$.

\section{APPLICATION TO ADAPTIVE STIMULATION OF PARKINSONIAN NEURONAL POPULATIONS}

All the results presented so far have been designed with neuroscience applications in mind. More precisely, in [7], a proportional stimulation strategy has been proposed to disrupt brain oscillations that are linked to Parkinsonian symptoms. This result was obtained based on a linearized version of the following model:

$$
\begin{aligned}
& \tau_{1} \dot{x}_{1}(t)=-x_{1}(t) \\
& \quad+S_{1}\left(c_{11} x_{1}\left(t-\delta_{11}\right)-c_{12} x_{2}\left(t-\delta_{12}\right)+\mu(t)+u_{1}(t)\right) \\
& \tau_{2} \dot{x}_{2}(t)=-x_{2}(t) \\
& \quad+S_{2}\left(c_{21} x_{1}\left(t-\delta_{21}\right)-c_{22} x_{2}\left(t-\delta_{22}\right)+u_{2}(t)\right) .
\end{aligned}
$$

This model was originally proposed in [19] (we recentered the model at 0 ) to explain a possible origin of parkinsonian brain oscillations in deep brain regions known as subthalamic nucleus (STN) and external globus pallidus (GPe). In this model, $x_{1}(t)$ and $x_{2}(t)$ represent the instantaneous activity of STN and GPe respectively, in number of spikes per second. $\tau_{1}, \tau_{2}>0$ are decay time constants. $S_{1}, S_{2}: \mathbb{R} \rightarrow \mathbb{R}$ are called the activation functions. For each $i, j \in\{1,2\}$, the constant $c_{i j} \geq 0$ represents the synaptic strength between $x_{j}$ and $x_{i}$ and $\delta_{i j} \geq 0$ describes the delay due to axonal propagation from $x_{j}$ to $x_{i} . u_{1}(t) \in \mathbb{R}$ models activity from the motor cortex, and $u_{2}(t) \in \mathbb{R}$ represents striatal activity. Finally, $\mu(t) \in \mathbb{R}$ represents the stimulation that can be introduced through implanted electrodes.

In the absence of stimulation $(\mu \equiv 0)$, this model exhibits sustained activity in the $\beta$-band $(13-30 \mathrm{~Hz})$ that arises from a too strong coupling between STN and GPe. These oscillations correlate with parkinsonian motor symptoms [9]. A spatio-temporal extension of this model was proposed and analyzed in [5], [4]. In those works, it was shown that a proportional stimulation strategy, relying on STN measurements only (meaning on $x_{1}(t)$ ) successfully disrupts these pathological oscillations provided that $c_{22}<1$, meaning that the GPe internal synaptic coupling is weak (which is in line with physiologic evidence).

\section{A. Theoretical analysis}

In practice, the parameters involved in (29) suffer high imprecision and variability. Hence, it would be desirable to adapt the value of the stimulation gain in real time. Since only $x_{1}(t)$ is available to measurements, we see (29) as a particular case of (11) with output $z(t):=x_{1}(t)$. We also let $\bar{\delta}:=\max _{i, j \in\{1,2\}} \delta_{i j}$. In line with the results of Section III, we propose the adaptive stimulation strategy

$$
\begin{aligned}
& \mu(t)=-\theta(t) x_{1}(t) \\
& \dot{\theta}(t)=\frac{1}{T}\left(\left|x_{1}(t)\right|-\varepsilon^{\prime} \theta(t)\right),
\end{aligned}
$$

where $\varepsilon^{\prime} \geq 0$ and $T>0$ denote tuning parameters. With this closed-loop stimulation policy, we have the following.

Theorem 4 (Adaptive brain stimulation) Assume $S_{1}, S_{2}$ : $\mathbb{R} \rightarrow \mathbb{R}$ are bounded Lipschitz nondecreasing functions satisfying $S_{1}(0)=S_{2}(0)=0$. Assume that the Lipschitz constant of $S_{2}$ is 1 and let $T>0$ be any constant parameter. Under the condition $c_{22}<1$ there exists $\gamma \in \mathcal{K}_{\infty}$ such that system (29) in closed loop with (30) satisfies, for all $\varepsilon \geq 0$, all $x_{0} \in \mathcal{C}^{2}$, and all bounded $u: \mathbb{R}_{\geq 0} \rightarrow \mathbb{R}^{2}$,

$$
\limsup _{t \rightarrow+\infty}|x(t)| \leq \gamma(\varepsilon+\|u\|) .
$$

The main steps of the proof are as follows. Assumption 3 is fulfilled due to the properties of the functions $S_{i}$. Assumption 4 can be checked by creating a LyapunovKrasovskii functional for (29) in a closed loop with $\mu(t)=$ $-\theta x_{1}(t)$, for some constant $\theta>0$, and in the absence of disturbances $(u \equiv 0)$. This is done along the proof steps of the main result in [4], which establishes the high-gain stabilizability of a spatio-temporal extension of (29). Finally, we use Theorem 3 to obtain robust stability. All initial states are considered because, due to the boundedness of $S_{i}$, every solution eventually enters the invariant set of Assumption 3.

Like the high-gain stimulation policies proposed in [7], [5], [4], the proposed adaptive stimulation successfully disrupts pathological oscillations in this model. As we detail in Section IV-B, the adaptive nature of this control law offers interesting advantages as compared to those policies, specifically in presence of uncertain or varying parameters.

The result is based on limited information on the model, namely the maximal slope of the activation function $S_{2}$ (normalized here to 1) and the GPe internal synaptic weight $c_{22}$. In particular, the proposed strategy is valid for any synaptic weights between STN and GPe and any transmission delays $\delta_{i j}$. Moreover, no measurement of GPe activity is needed.

\section{B. Simulation results}

We ran the simulations of system (29) in Python 2.7, using the pydelay library. The simulation parameters were chosen as follows: the time constants $\tau_{1}=6 \mathrm{~ms}, \tau_{2}=14$ $\mathrm{ms}$; the delays $\delta_{11}=0 \mathrm{~ms}, \delta_{12}=\delta_{21}=6 \mathrm{~ms}, \delta_{22}=4$ $\mathrm{ms}$; the connection strength coefficients $c_{11}=0, c_{12}=$ $20, c_{21}=10.7, c_{22}=0.2$. The activation functions $S_{i}$ were picked as $S_{i}(s)=m_{i} b_{i} /\left(b_{i}+\left(m_{i}-b_{i}\right) \exp (-4(s+\right.$ $\left.\left.\left.m_{i} \ln \left(\left(m_{i}-b_{i}\right) / b_{i}\right) / 4\right) / m_{i}\right)\right)-m_{i} / 2$, with $m_{1}=300 \mathrm{spk} / \mathrm{s}$, $m_{2}=400 \mathrm{spk} / \mathrm{s}, b_{1}=17 \mathrm{spk} / \mathrm{s}, b_{2}=75 \mathrm{spk} / \mathrm{s}$. All the above parameters, except $c_{22}$ (chosen small, to satisfy the stabilizability condition in Theorem 4), correspond to the parkinsonian state in [19]. The control parameters were 
picked as $\varepsilon^{\prime}=10^{-4}$ and $T=0.01 \mathrm{~ms}$. The disturbance $u_{1}$ was picked as a random noise drawn from a uniform distribution on $(-2 ; 2) . u_{2}$ was set at zero.

Figure 1 illustrates the efficacy of the proposed adaptive stimulation. For $t \in[0 ; 0.1]$, the feedback is off and the systems exhibits sustained oscillations. At time $t=0.1$, the stimulation is switched on. The adaptive gain $\theta$ increases (at a rate essentially given by $T$ ) and reaches a value that leads to significantly decreased oscillations (whose amplitude depends on the disturbance magnitude and the choice of $\varepsilon^{\prime}$ ). At $t=0.5 \mathrm{~s}$, the connection weights $c_{12}$ and $c_{21}$ were artificially diminished by a factor 2 , for which a weaker proportional gain is needed. Accordingly, $\theta$ automatically adjusts to a weaker value, giving rise to a stimulation signal $\mu$ of lower amplitude.
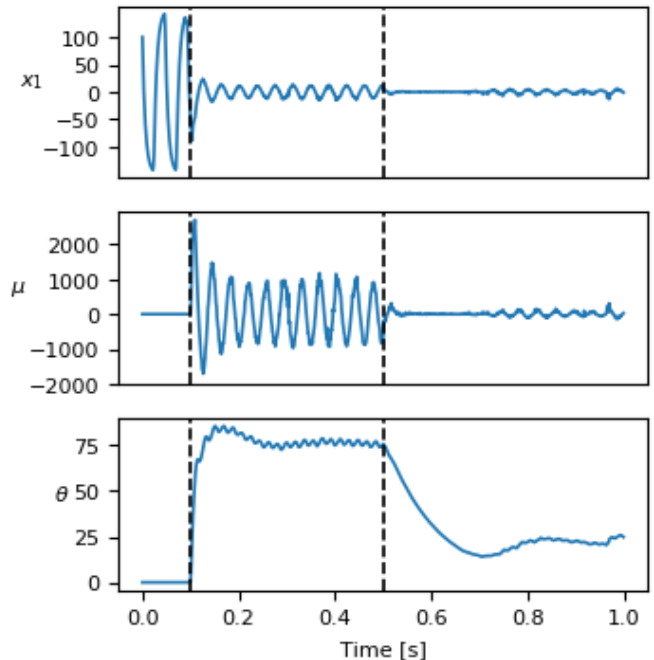

Fig. 1: Simulation results for the closed-loop adaptive system (29) in closed loop with (30). Top: STN activity $x_{1}(t)$. Middle: applied stimulation $\mu(t)=-\theta(t) x_{1}(t)$. Bottom: adaptive control gain $\theta(t)$.

Figure 2 represents the magnitude of steady-state oscillations of $x_{1}$ and $x_{2}$ as a function of the chosen parameter $\varepsilon^{\prime}$. In line with Theorem 4, this steady-state amplitude is an increasing function of $\varepsilon^{\prime}$ and vanishes for $\varepsilon^{\prime}=0$ with $u_{1}$ and $u_{2}$ set to zero.

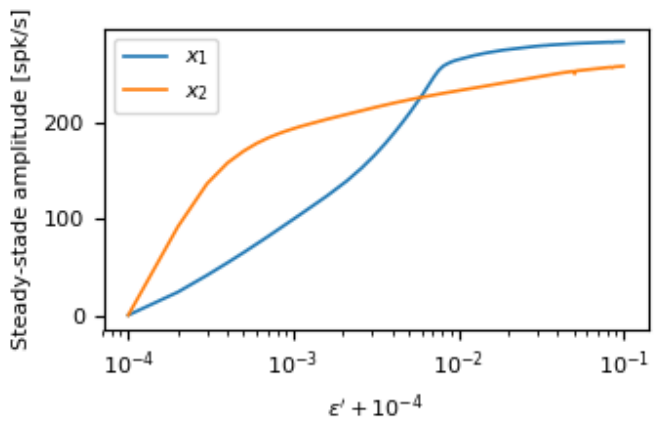

Fig. 2: Steady state amplitude $\left(\max \left\{x_{i}(t)\right\}-\min \left\{x_{i}(t)\right\}\right.$ for $\left.t \in[19,20] s\right)$ for different values of $\varepsilon^{\prime}:=\varepsilon T$. The remaining parameters were kept identical as in the simulation performed to obtain Figure 1 but with $u=0$. The results obtained are in accordance with Theorem 4 which states that the steady-state amplitude of the system is upper bounded by a $\mathcal{K}_{\infty}$ class function of $\varepsilon+\|u\|$. The $\mathrm{x}$-axis is shifted by $10^{-4}$ to allow displaying values for $\varepsilon^{\prime}=0$.

\section{CONCLUSIONS}

We conclude that the adaptive feedback explored here constitutes a promising approach to control pathological oscillations such as in Parkinson's disease or epilepsy. Such paradigms should be explored by future studies.

\section{REFERENCES}

[1] A. Benabid, P. Pollak, C. Gervason, D. Hoffmann, D. Gao, M. Hommel, J. Perret, and J. de Rougemont. Long-term suppression of tremor by chronic stimulation of the ventral intermediate thalamic nucleus. The Lancet, 337:403-406, 1991.

[2] C. Byrnes and A. Isidori. A frequency domain philosophy for nonlinear systems, with applications to stabilization and to adaptive control. In CDC84, pages 1569-1573, 1984.

[3] R. Carron, A. Chaillet, A. Filipchuk, W. Pasillas-Lépine, and C. Hammond. Closing the Loop of Deep Brain Stimulation. Frontiers in Systems Neuroscience, 13(112):1-18, 2013.

[4] A. Chaillet, G. Detorakis, S. Palfi, and S. Senova. Robust stabilization of delayed neural fields with partial measurement and actuation. Automatica, 83:262-274, Sep. 2017.

[5] G. Detorakis, A. Chaillet, S. Palfi, and S. Senova. Closed-loop stimulation of a delayed neural fields model of parkinsonian STNGPe network: a theoretical and computational study. Frontiers in Neuroscience, 9(237), 2015.

[6] R. Driver. Existence and stability of solutions of a delay-differential system. Arch. Rational Mech. Anal., 10(1):401-426, 1962.

[7] I. Haidar, W. Pasillas-Lépine, A. Chaillet, E. Panteley, S. Palfi, and S. Senova. A firing-rate regulation strategy for closed-loop deep brain stimulation. Biological Cybernetics, 110(1):55-71, 2016.

[8] J. Hale. Theory of functional differential equations. Springer-Verlag, New York-Heidelberg, 1977.

[9] C. Hammond, H. Bergman, and P. Brown. Pathological synchronization in Parkinson's disease: networks, models and treatments. Trends in Neurosciences, 30(7):357-364, 2007.

[10] A. Ilchmann and H. Logemann. Adaptive $\lambda$-tracking for a class of infinite-dimensional systems. Systems Control Lett., 34:11-21, 1998.

[11] A. Ilchmann and E. Ryan. Universal $\lambda$-tracking for non-linearlyperturbed systems in the presence of noise. Automatica, 30:337-346, 1994.

[12] P. A. Ioannou and P. V. Kokotovic. Instability analysis and improvement of robustness of adaptive control. Automatica, 20:583-594, 1984.

[13] H. Kankanamalage, Y. Lin, and Y. Wang. On Lyapunov-Krasovskii Characterizations of Input-to-Output Stability. Proceedings of the IFAC World Congress 2017, pages 1-6, July 2017.

[14] I. Karafyllis. The non-uniform in time small-gain theorem for a wide class of control systems with outputs. European Journal of Control, 10(4):307-323, 2004.

[15] I. Karafyllis, P. Pepe, and Z. Jiang. Global output stability for systems described by retarded functional differential equations: Lyapunov characterizations. European Journal of Control, 14(6):516-536, 2008.

[16] I. Karafyllis, P. Pepe, and Z. Jiang. Input-to-Output Stability for systems described by retarded functional differential equations. European Journal of Control, 14(6):539-555, 2008.

[17] V. Lakshmikantham and X. Z. Liu. Stability analysis in terms of two measures. World Scientific Publishing Co., Inc., River Edge, NJ, 1993.

[18] I. Mareels. A simple selftuning controller for stably invertible systems. Systems \& Control Letters, 4(1):5-16, feb 1984.

[19] A. Nevado-Holgado, J. Terry, and R. Bogacz. Conditions for the generation of beta oscillations in the subthalamic nucleus-globus pallidus network. Journal of Neuroscience, 30:12340-12352, 2010.

[20] R. Ortega and Y. Tang. Robustness of adaptive controllers - A survey. Automatica, 25:651-677, 1989.

[21] P. Pepe. On Liapunov-Krasovskii functionals under Carathéodory conditions. Automatica, 43(4):701-706, 2007.

[22] E. Sontag and Y. Wang. Notions of input-to-output stability. Systems \& Control Letters, 38:235-248, 1999.

[23] A. R. Teel and L. Praly. A smooth Lyapunov function from a class$\mathcal{K} \mathcal{L}$ estimate involving two positive semidefinite functions. ESAIM Control Optim. Calc. Var., 5:313-367, 2000.

[24] V. I. Vorotnikov. Partial stability and control. Birkhauser, 1998.

[25] N. Yeganefar, P. Pepe, and M. Dambrine. Input-to-state stability of time-delay systems: a link with exponential stability. IEEE Trans. Automat. Control, 53(6):1526-1531, 2008. 